\title{
New Perspective on the Conventional Solutions of the Nonlinear Time-Fractional Partial Differential Equations
}

\author{
Hijaz Ahmad (D, ${ }^{1}$ Ali Akgül, ${ }^{2}$ Tufail A. Khan, ${ }^{1}$ Predrag S. Stanimirović, ${ }^{3}$ \\ and Yu-Ming Chu ${ }^{40}{ }^{4,5}$ \\ ${ }^{1}$ Department of Basic Sciences, University of Engineering and Technology Peshawar, Peshawar, Pakistan \\ ${ }^{2}$ Art and Science Faculty, Department of Mathematics, Siirt University, Siirt TR-56100, Turkey \\ ${ }^{3}$ Faculty of Science and Mathematics, University of Niš, Višegradska 33, Niš 18000, Serbia \\ ${ }^{4}$ Department of Mathematics, Huzhou University, Huzhou 313000, China \\ ${ }^{5}$ Hunan Provincial Key Laboratory of Mathematical Modeling and Analysis in Engineering, \\ Changsha University of Science \& Technology, Changsha 410114, China
}

Correspondence should be addressed to Yu-Ming Chu; chuyuming2005@126.com

Received 4 September 2020; Revised 9 September 2020; Accepted 18 September 2020; Published 6 October 2020

Academic Editor: Mostafa M. A. Khater

Copyright (C) 2020 Hijaz Ahmad et al. This is an open access article distributed under the Creative Commons Attribution License, which permits unrestricted use, distribution, and reproduction in any medium, provided the original work is properly cited.

\begin{abstract}
The role of integer and noninteger order partial differential equations (PDE) is essential in applied sciences and engineering. Exact solutions of these equations are sometimes difficult to find. Therefore, it takes time to develop some numerical techniques to find accurate numerical solutions of these types of differential equations. This work aims to present a novel approach termed as fractional iteration algorithm-I for finding the numerical solution of nonlinear noninteger order partial differential equations. The proposed approach is developed and tested on nonlinear fractional-order Fornberg-Whitham equation and employed without using any transformation, Adomian polynomials, small perturbation, discretization, or linearization. The fractional derivatives are taken in the Caputo sense. To assess the efficiency and precision of the suggested method, the tabulated numerical results are compared with the standard variational iteration method and the exact solution as well. In addition, numerical results for different cases of the fractional-order $\alpha$ are presented graphically, which show the effectiveness of the proposed procedure and revealed that the proposed scheme is very effective, suitable for fractional PDEs, and may be viewed as a generalization of the existing methods for solving integer and noninteger order differential equations.
\end{abstract}

\section{Introduction}

The nonlinear PDEs have become a hot topic in the field of nonlinear science, which has been used to describe the problems in many fields, such as quantum mechanics, image processing, ecology and economic system, and epidemiology. PDEs are broadly emerging in different physical applications like dispersing and propagation of waves, magnetic resonance imaging, computational fluid dynamics, magnetohydrodynamic move through pipes, phenomena of supersonic and turbulence flow, acoustic transmission, and traffic. More details can be found in [1] and the references therein. PDEs are used in population models, medical imaging, proper distribution of oxygen to the healing tissues, electrical signalling of nerves, etc. [2]. The popularity of PDE has been confirmed in a very actual prediction of the number of COVID cases $[3,4]$. Using PDE, it is possible to make a model of the shape of COVID-19 [5]. However, for some complex problems in these fields, the fractional PDE is more accurate than integer-order partial differential equation. So, generating numerical solutions of fractional PDEs has become extremely important.

The noninteger order Calculus was created immediately after the integer order Calculus, which means that it dates back to the $17^{\text {th }}$ century. The notation $\left(\mathrm{d}^{i} f(\psi) / \mathrm{d} \psi^{i}\right)$ was originated around 1675 by Leibniz to represent the $i$ th derivative of a function $f(\psi)$, assuming integer values of $i$. In $1695, \mathrm{~L}^{\prime}$ Hospital stated the problem: "What if $i$ is $(1 / 2)$ ?" 
Later, Leibniz used fractional derivatives (FD) symbols in his research. Accordingly, the concept of an FD appeared almost at the same time as the integer order derivative. Many famous researchers in the $18^{\text {th }}$ and $19^{\text {th }}$ century, for example Euler, Lagrange, Laplace, Fourier, and many others, contributed to the development of the fractional calculus (FC). The first problem modeled in terms of FC was the known problem of the tautochrone curve. In 1823, Abel found a solution in terms of an integral equation, which is based on the Riemann-Liouville definition of the fractional integration. However, the popularity of fractional partial differential equations (FPDE) was slowed down by some incomplete and conflicting definitions and difficulties in establishing consistent regulations for inverse operations. Satisfactory definitions for the integration and differentiation with fractional power were developed in the middle of the $19^{\text {th }}$ century. FC is still an active of research in engineering and sciences [6-10].

Due to the importance of numerical solution of fractional PDEs (FPDEs) in science and engineering, some powerful numerical techniques are developed in the literature, and many prominent researchers have made contributions in this area. These include finite element methods [11], mixed finite element (MFE) methods [12], (local) discontinuous Galerkin methods [13], finite volume methods [14], Jacobi collocation [15], variational iteration method [16], generalized Kudryashov method [17], finite Hankel transform procedure [18], modified Khater method [19], residual power series method [20], modified auxiliary equation method [21], local meshless method [22], RBF collocation method [23], operational matrix of B-spline functions [24], and some other advanced numerical methods as well. Zhang and $\mathrm{Xu}$ [25], Lin et al. [26] considered some spectral approximations for the time-fractional water wave model, time-fractional diffusion equation. Most nonlinear TFPDEs do not have accurate exact solutions. Therefore, direct and iterative approaches are applied. Most of the methods for nonlinear TFPDEs give infinite series solutions or mesh-based method. These methods are time-consuming due to repeated calculation in series solution and mesh creation in mesh-based methods.

Opposite to great popularity and applicability, the shortcoming is obvious in the available numerical techniques of reasonably high orders for finding approximate solutions of FPDEs. Such a need for a general method, which is usable in solving linear, nonlinear, homogenous, nonhomogeneous, and multivariable FPDEs problems without major changes, is the inspiration for the current research. Recently, many scholars have investigated the numerical solutions of the fractional PDEs, which has effectively promoted the advancement of the field of nonlinear PDEs. However, in general, numerical techniques have some limitations such as low accuracy, mesh generation, transformations, stability, convergence, and difficulty of implementing in complex geometries. In recent years, the variational iteration methods (VIM) have become popular in the field of numerical approximations. In this technique, the discretization of the domain and linearization of given differential equations is not required. We simply need to calculate the Lagrange multiplier of the given differential equation by restricting the nonlinear terms and in series form analytical solution of the given differential equations can be obtained. VIM can be implemented in the easiest way and is more flexible than other techniques available in the literature. It was applied for the first time to FPDEs by $\mathrm{He}$ in [27], and later on by Odibat and Momani in [28]. Inc [29] employed it for the numerical approximation of fractional Burgers equations. Yulita et al. [30] applied VIM for the analytical treatment of fractional Zakharov-Kuznetsov equations, while Safari et al. [31] utilized it for fractional KdV Burger's Kuramoto equation. Das in [32] investigated fractional diffusion equations by using VIM and obtained the exact solution.

In this study, we present a novel approach, termed as fractional iteration algorithm-I, for solving noninteger order differential equations. The proposed approach is developed and tested on nonlinear fractional-order FornbergWhitham equation. This equation is defined as

$$
\frac{\partial \mathcal{U}^{\alpha}}{\partial t^{\alpha}}+\frac{\partial \mathcal{U}}{\partial \psi}-\frac{\partial \mathcal{U}^{3}}{\partial \psi^{2} \partial t}+\mathcal{U} \frac{\partial \mathcal{U}}{\partial \psi}=3 \frac{\partial \mathcal{U}}{\partial \psi} \frac{\partial^{2} \mathcal{U}}{\partial \psi^{2}}+\mathcal{U} \frac{\partial^{3} \mathcal{U}}{\partial \psi^{3}}, \quad t>0,0<\alpha \leq 1,
$$

subject to the beneath initial condition

$$
\mathcal{U}(\psi, 0)=\frac{4}{3} e^{(\psi / 2)} .
$$

The constant $\alpha$ in 1 lies in the interval $(0,1], \mathscr{U}(\psi, t)$ is the fluid velocity, $\psi$ is the spatial, and $t$ is the time coordinate.

The numerical solution of the Fornberg-Whitham type equations (1) is numerically challenging. To solve these equations numerically, several methods were proposed in the literature. Lu in [33] solved Fornberg-Whitham type equations by He's variational iteration method. The approximate analytical solutions to this type of equations were presented by Abidi and Omrani in [34] employing homotopy analysis technique. Zhou and Tian [35] obtained a special type of travelling wave solution of the FornbergWhitham type equations by applying the bifurcation method. Yin et al. [36] classified all the travelling solutions obtained by an improved qualitative method of Fornberg-Whitham equation. Feng and $\mathrm{Wu}$ [37] reduced this equation to simple ODE and solved it by factorization technique successfully. Jiang and Bi [38] presented the bifurcation method and smooth travelling wave solutions are obtained. Reduced differential transform method has been utilized by Hesam [39] for this type of equations and in convergent power series, the results are obtained. Biazar and Eslami [40] used He's HPM for the analytical solution of equation (1). Three different types of Fornberg-Whitham equation were investigated by Boutarfa et al. [41] who used the reproducing kernel Hilbert space method. A novel analytical approach for solving fractional Fornberg-Whitham equation was proposed in [42]. An iterative method for finding approximate analytical solutions to the fractional Fornberg-Whitham equation was proposed in [43]. An analytical solution of Fornberg-Whitham type equations in 
view of the fractional Caputo operator was considered in [44]. Our aim in this work is to apply the MVIA-I for these two types of Fornberg-Whitham type equations.

The remaining sections of the paper are organized on the basis of the following organization. In Section 2, some special and basic functions of fractional Calculus are defined. In Section 3, the fractional iteration algorithm-I is explained. Its convergence analysis is discussed in Section 4, and its implementation is illustrated for nonlinear FornbergWhitham type equations in Section 5. In Section 6, the results are discussed, and some utilizations of the proposed scheme are given. Conclusion is explained in the last Section 7.

\section{Basic Functions and Properties of Fractional Calculus}

This section aims to present some basic definitions and notions, which have significant importance in the FC. First of all, we discuss some basic special functions. Basic definitions of fractional derivatives were introduced thereafter.

2.1. Special Functions. In fractional/fractal calculus, the gamma, Beta, and Mittag-Leffler functions are the fundamental and key tools to understand the origin of its computational challenges.

2.1.1. Gamma Function. The gamma function $\Gamma(z)$ is defined by the Euler integral of the second kind [45]:

$$
\Gamma(z)=\int_{0}^{\infty} e^{-t} t^{z-1} \mathrm{~d} t .
$$

This integral is convergent in the right half of the complex plane $\operatorname{re}(z)>0$.

2.1.2. Beta Function. The Beta function $\beta(z, w)$ is defined by the Euler integral of the first kind [45]:

$$
\beta(z, w)=\int_{0}^{\infty}(1-t)^{w-1} t^{z-1} \mathrm{~d} t, \quad \operatorname{re}(z)>0, \quad \operatorname{re}(w)>0 .
$$

The relationship between Beta function and Gamma function can be established as

$$
\beta(z, w)=\frac{\Gamma(w) \Gamma(z)}{\Gamma(w+z)} .
$$

2.1.3. Mittag-Leffler Function. We describe the one-parameter and two-parameter Mittag-Leffler function, respectively, as [46]

$$
\begin{aligned}
E_{\alpha}(z) & =\sum_{k=0}^{\infty} \frac{z^{k}}{\Gamma(\alpha k+1)}, \\
E_{\alpha, \beta}(z) & =\sum_{k=0}^{\infty} \frac{z^{k}}{\Gamma(\alpha k+\beta)} .
\end{aligned}
$$

2.2. Different Definitions of Fractional Derivatives. We present some main definitions of the fractional derivatives as follows:

Definition 1. The right-modified Riemann-Liouville derivative is defined as [47]

$$
\frac{\partial^{\alpha} \mathscr{U}(\psi, t)}{\partial t^{\alpha}}=\frac{1}{\Gamma(1-\alpha)} \frac{\mathrm{d}}{\mathrm{d} t} \int_{t}^{T} \frac{(\mathcal{U}(\psi, \vartheta)-\mathcal{U}(\psi, t))}{(\vartheta-t)^{\alpha}} \mathrm{d} \vartheta, \quad 0<\alpha<1 .
$$

Definition 2. The Caputo fractional derivative is defined as [48]

$$
\frac{\partial^{\alpha} \mathscr{U}(\psi, t)}{\partial t^{\alpha}}=\frac{1}{\Gamma(1-\alpha)} \int_{0}^{t} \frac{\partial \mathscr{U}(\psi, \vartheta)}{\partial \vartheta}(t-\vartheta)^{-\alpha} \mathrm{d} \vartheta, \quad 0<\alpha<1 .
$$

Definition 3. The $\mathrm{ABC}$ derivative is introduced as [49]

$$
\mathrm{ABC}_{a} \frac{\partial^{\alpha} \mathscr{U}(\psi, t)}{\partial t^{\alpha}}=\frac{\mathrm{AB}(\alpha)}{1-\alpha} \int_{a}^{t} \mathscr{U}(\psi, x) E_{\alpha}\left(-\frac{\alpha(t-x)^{\alpha}}{1-\alpha}\right) \mathrm{d} x, \quad 0<\alpha<1,
$$

where $\mathrm{AB}(\alpha)$ is the normalization function.

Definition 4. The Ji-Huan He's fractional derivative is defined by the rule [50]

$$
\frac{\partial^{\alpha} \mathscr{U}(\psi, t)}{\partial t^{\alpha}}=\frac{1}{\Gamma(1-\alpha)} \frac{\mathrm{d}}{\mathrm{d} x} \int_{t_{0}}^{t}(t-\vartheta)^{-\alpha}\left[\mathscr{U}_{0}(\vartheta)-\mathscr{U}(\vartheta)\right], \quad 0<\alpha<1 .
$$

Definition 5. The Ji-Huan He's fractal derivative is defined as [50]

$$
\frac{\partial \mathscr{U}}{\partial \psi^{\alpha}}=\Gamma(1-\alpha) \lim _{\Delta \psi=\psi_{1}-\psi_{2} \rightarrow L} \frac{\mathscr{U}\left(\psi_{1}\right)-\mathscr{U}\left(\psi_{2}\right)}{\left(\psi_{1}-\psi_{2}\right)^{\alpha}} .
$$

Definition 6. The Grünwald-Letnikov fractional derivative is presented as [45] 


$$
D_{t}^{\alpha} f(t)=\lim _{h \longrightarrow 0} \frac{1}{h^{\alpha}} \sum_{k=0}^{m}(-1)^{k}\left(\begin{array}{l}
\alpha \\
k
\end{array}\right) f(t-k h), \quad \psi-1<\alpha<\psi .
$$

\section{Fractional Iteration Algorithm-I}

In this section, the main idea of the fractional iteration algorithm-I is illustrated by considering a nonlinear differential equation of the generic form

$$
L[\mathscr{U}(\psi)]+N[\mathscr{U}(\psi)]=g(\psi),
$$

where $L[\mathcal{U}(\psi)]$ and $N[\mathcal{U}(\psi)]$ denote linear and nonlinear operators, respectively, whereas $g(\psi)$ is a nonhomogeneous term. For an appropriate given initial condition $\mathscr{U}_{0}(\psi)$, series $\mathcal{U}_{k+1}(\psi)$, which approximates the solution of equation (13), can be obtained as

$$
\mathscr{U}_{k+1}(\psi)=\mathscr{U}_{k}(\psi)+\rho \int_{0}^{\psi} \lambda(\vartheta)\left[L\left\{\mathscr{U}_{k}(\vartheta)\right\}+\widetilde{N\left\{\mathscr{U}_{k}(\vartheta)\right\}}-g(\vartheta)\right] \mathrm{d} \vartheta,
$$

where $\overline{\mathcal{U}_{k}(\vartheta)}$ is a restricted term, which gives $\delta \overline{\mathcal{U}_{k}(\vartheta)}=0$ with respect to the variation $\delta$, and $\rho$ and $\lambda(\vartheta)$ are an auxiliary parameter and Lagrange multiplier, respectively, which can be optimally determined. The first one is used to accelerate the convergence to the exact solution [51-55], while the second one is used to construct the correction function [56].

A suitable value of $\lambda(\vartheta)$ can be achieved applying $\delta$ on both sides of recurrent relation (14) with respect to $\mathscr{U}_{k}(\psi)$, which leads to

$$
\delta \mathscr{U}_{n+1}(\psi)=\delta \mathscr{U}_{n}(\psi)+\rho \delta \int_{0}^{\psi} \lambda(\vartheta)\left[L\left\{\mathscr{U}_{k}(\vartheta)\right\}+\widetilde{N\left\{\mathscr{U}_{k}(\vartheta)\right\}}-g(\vartheta)\right] \mathrm{d} \vartheta .
$$

For nonlinear problems, the nonlinear terms have to be considered as restricted variations for obtaining the value of Lagrange multiplier, and a correction functional can be easily constructed after determining the identified value of corresponding nonlinear terms. The beneath Lagrange multipliers can be obtained in the following way:

$$
\begin{aligned}
& \lambda=-1, \quad \text { for } i=1, \\
& \lambda=\psi-t, \quad \text { for } i=2 .
\end{aligned}
$$

Also, the following general formula for the Lagrange multiplier in the cases $i \geq 1$ is available:

$$
\lambda=\frac{(-1)^{i}(\vartheta-t)^{i-1}}{(i-1) !} .
$$

After finding the value of $\widetilde{\mathcal{U}_{k}(\vartheta)}$, an iteration formula is constructed by using this value in the corrective function (14) as follows:

$$
\mathscr{U}_{n+1}(\psi)=\mathscr{U}_{n}(\psi)+\rho \int_{0}^{\psi} \frac{(-1)^{i}(\vartheta-t)^{i-1}}{(i-1) !}\left[L\left\{\mathscr{U}_{k}(\vartheta)\right\}+N\left\{\mathscr{U}_{k}(\vartheta)\right\}-g(\vartheta)\right] \mathrm{d} \vartheta .
$$

The iterative sequence $\mathcal{U}_{k}$ can be obtained starting from a proper initial approximation and using the iterative formula (18). It is convenient to repeat iterations many times to arrive at the given accuracy for the advanced computer technique. An exact solution $\mathcal{U}(\psi)$ is obtained as the limiting value

$$
\mathscr{U}(\psi)=\lim _{k \longrightarrow \infty} \mathscr{U}_{k}(\psi)
$$

It is worth mentioning that the proposed algorithm may be considered as a nice refinement in existing analytical and numerical methods, where the discretization, transformations, and linearization are not required, and the numerical solution of the given differential equations can be obtained in series form as

$$
\left\{\begin{array}{l}
\mathscr{U}_{0}(\psi) \text { is an appropriate initial approximation, } \\
\mathcal{U}_{1}(\psi, \rho)=\mathscr{U}_{0}(\psi)+\rho \int_{0}^{\psi} \lambda(\vartheta)\left[L\left\{\mathscr{U}_{0}(\vartheta)\right\}+N\left\{\mathcal{U}_{0}(\vartheta)\right\}-g(\vartheta)\right] \mathrm{d} \vartheta \\
\mathcal{U}_{k+1}(\psi, \rho)=\mathscr{U}_{0}(\psi, \rho)+\rho \int_{0}^{\psi} \lambda(\vartheta)\left[N\left\{\mathcal{U}_{k}(\vartheta, \rho)\right\}-g(\vartheta, \rho)\right] \mathrm{d} \vartheta, \\
k=1,2, \ldots
\end{array}\right.
$$

We employ this procedure for finding the analytical/ numerical solution of nonlinear fractional-order Fornberg-Whitham equations. When $\rho=1$, this procedure given in equation (20) becomes the standard variational iteration algorithm-I. Equation (20) has two obvious advantages; one is the limited step, which is needed for better 
accuracy, while the other is an auxiliary parameter $(\rho)$, which ensures the convergence, and a more accurate solution can be gained after a higher iteration process.

\section{Convergence Analysis}

The convergence of the algorithm proposed in Section 3 for solving nonlinear fractional partial differential equations will be examined in this section. This algorithm can be performed in an effective and trustworthy way and can handle fractional differential equation (13) as well. When the fractional iteration algorithm is implemented for the numerical investigation of the fractional-order FornbergWhitham equation, the linear operator $L$ is defined as $L=\left(\partial^{\alpha} / \partial t^{\alpha}\right)$. First, in (21), we define the operator $R$ for solving problems of such type:

$$
R \mathscr{U}(\psi, t, \rho):=\rho \int_{0}^{t} \lambda(\vartheta)[L \mathscr{U}(\psi, \vartheta, \rho)+\widetilde{N \mathscr{U}(\psi, \vartheta, \rho)}-g(\vartheta)] \mathrm{d} \vartheta
$$

where $k \geq 0$ and $w_{k}$ and $v_{k}$ are defined by

$$
\begin{aligned}
& \left\{\begin{array}{l}
w_{0}(\psi, t)=\mathscr{U}_{0}(\psi, t), \\
v_{0}(\psi, t)=w_{0}(\psi, t),
\end{array}\right. \\
& \left\{\begin{array}{l}
w_{1}(\psi, t, \rho)=R v_{0}(\psi, t), \\
v_{1}(\psi, t, \rho)=v_{0}(\psi, t)+R v_{0}(\psi, t),
\end{array}\right. \\
& \left\{\begin{array}{l}
\mathcal{U}_{1}(\psi, t, \rho)=R v_{0}(\psi, t), \\
v_{1}(\psi, t, \rho)=v_{0}(\psi, t)+w_{1}(\psi, t, \rho),
\end{array}\right. \\
& \left\{\begin{array}{l}
W_{k+1}(\psi, t, \rho)=R v_{n}(\psi, t, \rho), \\
v_{n+1}(\psi, t, \rho)=v_{n}(\psi, t, \rho)+R v_{n}(\psi, t, \rho) .
\end{array}\right.
\end{aligned}
$$

In general, for $k \geq 1$, it can be written as

$$
\left\{\begin{array}{l}
\mathcal{U}_{k+1}(\psi, t, \rho)=R v_{k}(\psi, t, \rho), \\
v_{k+1}(\psi, t, \rho)=v_{k}(\psi, t, \rho)+W_{k+1}(\psi, t, \rho) .
\end{array}\right.
$$

Accordingly,

$$
\mathcal{U}(\psi, t, \rho):=\lim _{k \longrightarrow \infty} v_{k}(\psi, t, \rho)=w_{0}(\psi, t)+\sum_{k=1}^{\infty} w_{k}(\psi, t, \rho) .
$$

The initial iteration $\mathscr{U}_{0}(\psi, t)$ can be chosen uninhibitedly, but it needs to fulfil the corresponding initial-boundary conditions. The determination of appropriate initial approximation will give productive and accurate results. The $n^{\text {th }}$-order truncated series $\mathcal{U}_{n}(\psi, t, \rho):=w_{0}(\psi, t)+$ $\sum_{k=1}^{n} w_{k}(\psi, t, \rho)$ can be used to approximate the solution. The unknown parameter $\rho$ in $\mathscr{U}_{n}(\psi, t, \rho)$ ensures that the hypothesis is fulfilled by utilizing 2 -norm error of the residual function. The error analysis and convergence criteria of VIA-I with an auxiliary parameter are revealed using the following theorems $[57,58]$.

Theorem 1. The operator $R$ defined in (21) maps a Hilbert space $H$ to $H$. The solution given in (24) can be given in the following form of series:

$$
\mathcal{U}(\psi, t):=\lim _{k \longrightarrow \infty} v_{k}(\psi, t, \rho)=w_{0}(\psi, t)+\sum_{k=1}^{\infty} w_{k}(\psi, t, \rho)
$$

It converges if $\exists \rho \neq 0,0<\beta<1$, such that

$$
\left\{\begin{array}{l}
\left\|R v_{0}(\psi, t)\right\| \leq \beta\left\|v_{0}(\psi, t)\right\|, \\
\left\|R v_{1}(\psi, t, \rho)\right\| \leq \beta\left\|R v_{0}(\psi, t)\right\|, \\
\left\|R v_{k}(\psi, t, \rho)\right\| \leq \beta\left\|R v_{k-1}(\psi, t, \rho)\right\|, \quad k=2,3,4, \ldots .
\end{array}\right.
$$

Lemma 1. Let $Q$ be a function from a Hilbert space $H$ to $H$, and the operator $L$ required in (13) be defined as $L=\left(\partial^{i} / \partial t^{i}\right), i=1,2$ and the Lagrange multiplier be defined optimally by the variation theory, then

$$
\left\{L \int_{0}^{t} \lambda(\vartheta) Q(\psi, \vartheta, \rho) \mathrm{d} \vartheta\right\}=-Q(\psi, \vartheta, \rho) .
$$

Theorem 2. Let the operator $L$ needed in 14 be defined as $L=\left(\partial^{i} / \partial t^{i}\right), i=1,2$. If we have the series solution (24) defined by

$$
\mathcal{U}(\psi, t):=w_{0}(\psi, t)+\sum_{k=1}^{\infty} w_{k}(\psi, t, \rho),
$$

then $\mathcal{U}(\psi, t)$ is an exact solution to the nonlinear partial differential equation (13).

Theorem 3. Let us suppose that the solution $\mathcal{U}(\psi, t):=w_{0}(\psi, t)+\sum_{n=1}^{\infty} w_{n}(\psi, t, \rho)$, given in (24), converges to the exact solution of the model equation (1). Also, assume that if the approximate solution is the truncated series $\mathcal{U}_{N}(\psi, t):=w_{0}(\psi, t)+\sum_{k=1}^{N} w_{k}(\psi, t, \rho)$ then the maximum error norm can be assessed as

$$
\left\|\mathcal{U}(\psi, t)-\mathcal{U}_{N}(\psi, t)\right\| \leq\left\|\frac{1}{1-\beta} \beta^{N+1}\right\| \| w_{0} .
$$

\section{Implementation of the Algorithm and Examples}

In this section, to clarify step by step solution procedure of the fractional iteration algorithm-I, the following timefractional Fornberg-Whitham equation (1) is considered:

$$
\frac{\partial \mathcal{U}^{\alpha}}{\partial t^{\alpha}}+\frac{\partial \mathcal{U}}{\partial \psi}-\frac{\partial \mathcal{U}^{3}}{\partial \psi^{2} \partial t}+\mathcal{U} \frac{\partial \mathcal{U}}{\partial \psi}=3 \frac{\partial \mathcal{U}}{\partial \psi} \frac{\partial^{2} \mathcal{U}}{\partial \psi^{2}}+\mathcal{U} \frac{\partial^{3} \mathcal{U}}{\partial \psi^{3}}, \quad t>0, \quad 0<\alpha \leq 1 .
$$

The initial condition is

$$
\mathcal{U}(\psi, 0)=\frac{4}{3} e^{(x / 2)},
$$

and the exact solution taken from [41] is equal to 


$$
\mathcal{U}(\psi, t)=\frac{4}{3} e^{(x / 2)-(2 t / 3)}
$$

Numerical solution $\boldsymbol{U}_{k+1}(\psi)$ of equation (13) for the provided initial condition $\mathscr{U}_{0}(\psi)$ can be achieved by means of

$$
\begin{aligned}
& \mathscr{U}_{k+1}(\psi, t, \rho)=\mathscr{U}_{k}(\psi, t, \rho)+\rho \int_{0}^{t} \lambda(\vartheta)\left[\frac{\partial \mathcal{U}_{k}^{\alpha}(\psi, \vartheta, \rho)}{\partial(\vartheta)^{\alpha}}-\overline{\frac{\partial^{3} \mathcal{U}_{k}(\psi, \vartheta, \rho)}{\partial(\psi)^{2} \partial(\vartheta)}}+\frac{\partial \mathscr{U}_{k}(\psi, \vartheta, \rho)}{\partial(\psi)}+\overline{\mathscr{U}_{k}(\psi, \vartheta, \rho) \frac{\partial \mathcal{U}_{k}(\psi, \vartheta, \rho)}{\partial(\psi)}}\right. \\
& -3 \overline{\frac{\partial \mathcal{U}_{k}(\psi, \vartheta, \rho)}{\partial(\psi)} \frac{\partial^{2} U_{k}(\psi, \vartheta, \rho)}{\partial(\psi)^{2}}}-\overbrace{\mathcal{U}_{k}(\psi, \vartheta, \rho) \frac{\partial^{3} U_{k}(\psi, \vartheta, \rho)}{\partial(\psi)^{3}}}^{3}] \mathrm{d} \vartheta
\end{aligned}
$$

The Lagrange multiplier $\rho$ can be obtained using the variation theory. Multiplying both the sides of the equation (33) by $\delta$, one obtains

$$
\begin{aligned}
& \delta \mathscr{U}_{k+1}(\psi, t, \rho)=\delta \mathscr{U}_{k}(\psi, t, \rho)+\rho \delta \int_{0}^{t} \lambda(\vartheta)[\frac{\partial \mathscr{U}_{k}^{\alpha}(\psi, \vartheta, \rho)}{\partial(\vartheta)^{\alpha}}-\overbrace{\frac{\partial^{3} \mathscr{U}_{k}(\psi, \vartheta, \rho)}{\partial(\psi)^{2} \partial(\vartheta)}}+\frac{\partial \mathscr{U}_{k}(\psi, \vartheta, \rho)}{\partial(\psi)}+\overbrace{\mathscr{U}_{k}(\psi, \vartheta, \rho) \frac{\partial \mathcal{U}_{k}(\psi, \vartheta, \rho)}{\partial(\psi)}} \\
& -3 \overbrace{\frac{\partial \mathcal{U}_{k}(\psi, \vartheta, \rho)}{\partial(\psi)} \frac{\partial^{2} \mathcal{U}_{k}(\psi, \vartheta, \rho)}{\partial(\psi)^{2}}}-\overbrace{\mathcal{U}_{k}(\psi, \vartheta, \rho) \frac{\partial^{3} \mathcal{U}_{k}(\psi, \vartheta, \rho)}{\partial(\psi)^{3}}}] \mathrm{d} \vartheta .
\end{aligned}
$$

For nonlinear problems, the nonlinear terms are considered to be restricted variations for obtaining the value of Lagrange multiplier; i.e., $\sim \mathcal{U}_{k}(\psi, \vartheta, \rho)$ is a restricted term. This implies $\delta \sim \mathcal{U}_{k}(\psi, \vartheta, \rho)=0$ and gives the beneath value of the Lagrange multiplier $\lambda=-1$. After using the value of $\lambda(\vartheta)$ in the recurrence relation (34), the beneath recurrent dependence is obtained:

$$
\begin{aligned}
\mathscr{U}_{k+1}(\psi, t, \rho)= & \mathscr{U}_{k}(\psi, t, \rho)-\rho \int_{0}^{t}\left[\frac{\partial \mathcal{U}_{k}^{\alpha}(\psi, \vartheta, \rho)}{\partial(\vartheta)^{\alpha}}-\frac{\partial^{3} \mathscr{U}_{k}(\psi, \vartheta, \rho)}{\partial(\psi)^{2} \partial(\vartheta)}+\frac{\partial \mathscr{U}_{k}(\psi, \vartheta, \rho)}{\partial(\psi)}+\mathscr{U}_{k}(\psi, \vartheta, \rho) \frac{\partial \mathscr{U}_{k}(\psi, \vartheta, \rho)}{\partial(\psi)}\right. \\
& \left.-3 \frac{\partial \mathcal{U}_{k}(\psi, \vartheta, \rho)}{\partial(\psi)} \frac{\partial^{2} \mathscr{U}_{k}(\psi, \vartheta, \rho)}{\partial(\psi)^{2}}-\mathscr{U}_{k}(\psi, \vartheta, \rho) \frac{\partial^{3} \mathscr{U}_{k}(\psi, \vartheta, \rho)}{\partial(\psi)^{3}}\right] \mathrm{d} \vartheta
\end{aligned}
$$

Starting with a proper initial approximation and using the iterative formula (35), values in other iterations can be obtained. We stop the process at the third iteration. A residual function used before for variational iteration algorithm-II in [59] can be defined here for approximated solution to get optimal value of unknown parameter:

$$
\begin{aligned}
& r_{3}(\psi, t, \rho)=\frac{\partial \mathcal{U}_{3}^{\alpha}(\psi, t, \rho)}{\partial(t)^{\alpha}}-\frac{\partial^{3} \mathscr{U}_{3}(\psi, t, \rho)}{\partial(\psi)^{2} \partial(t)}+\frac{\partial \mathscr{U}_{3}(\psi, t, \rho)}{\partial(\psi)}+\mathscr{U}_{3}(\psi, t, \rho) \frac{\partial \mathscr{U}_{3}(\psi, t, \rho)}{\partial(\psi)} \\
& -3 \frac{\partial \mathcal{U}_{3}(\psi, t, \rho)}{\partial(\psi)} \frac{\partial^{2} \mathscr{U}_{3}(\psi, t, \rho)}{\partial(\psi)^{2}}-\mathscr{U}_{3}(\psi, t, \rho) \frac{\partial^{3} \mathscr{U}_{3}(\psi, t, \rho)}{\partial(\psi)^{3}} . \\
& e_{3}(\rho)=\left(\int_{0}^{1} \int_{0}^{1}\left|r_{3}(\psi, t, \rho)\right|^{2} \mathrm{~d} t \mathrm{~d} x\right)^{(1 / 2)} .
\end{aligned}
$$

And error of norm 2 of the above residual function with respect to the parameter $\rho$ for $(\psi, t) \in[a, b] \times[a, b]$ can be defined as 
TABLE 1: Comparison of exact and approximate solutions and absolute errors for equation (1).

\begin{tabular}{|c|c|c|c|c|c|c|}
\hline \multirow{3}{*}{$\psi$} & \multirow{3}{*}{$t$} & \multirow{3}{*}{ Exact solution } & \multicolumn{4}{|c|}{$\alpha=1$} \\
\hline & & & \multicolumn{2}{|c|}{ Approximate solutions } & \multicolumn{2}{|c|}{ Absolute errors } \\
\hline & & & Present & {$[60]$} & Present & {$[60]$} \\
\hline \multirow{2}{*}{0.5} & 0.5 & 1.22672588 & 1.226773083 & 1.22606593 & $4.7203 \times 10^{-5}$ & $7.0715 \times 10^{-4}$ \\
\hline & 1.0 & 0.87898750 & 0.8793454867 & 0.87385063 & $3.5798 \times 10^{-4}$ & $5.1368 \times 10^{-3}$ \\
\hline \multirow{2}{*}{1.0} & 0.5 & 1.57514721 & 1.57520781827 & 1.57429982 & $6.0608 \times 10^{-5}$ & $8.4739 \times 10^{-4}$ \\
\hline & 1.0 & 1.12864229 & 1.12910195442 & 1.12204642 & $4.5966 \times 10^{-4}$ & $6.5958 \times 10^{-3}$ \\
\hline \multirow{2}{*}{1.5} & 0.5 & 2.02252906 & 2.022606876 & 2.02144098 & $7.7816 \times 10^{-5}$ & $1.0880 \times 10^{-3}$ \\
\hline & 1.0 & 1.44920539 & 1.449795608 & 1.44073612 & $5.9021 \times 10^{-4}$ & $8.4692 \times 10^{-3}$ \\
\hline
\end{tabular}

TABLe 2: Approximate solutions for different values of $\alpha$ for equation (1).

\begin{tabular}{|c|c|c|c|c|c|c|c|}
\hline \multirow{2}{*}{$\psi$} & \multirow{2}{*}{$t$} & \multicolumn{2}{|c|}{$\alpha=0.9$} & \multicolumn{2}{|c|}{$\alpha=0.8$} & \multicolumn{2}{|c|}{$\alpha=0.7$} \\
\hline & & Present & {$[60]$} & Present & {$[60]$} & Present & {$[60]$} \\
\hline \multirow[b]{2}{*}{0.5} & 0.5 & 1.168447201 & 1.16963576 & 1.104821658 & 1.10867258 & 1.024679713 & 1.04366140 \\
\hline & 1.0 & 0.882003595 & 0.85834430 & 0.913371082 & 0.83877324 & 0.937152668 & 0.81199739 \\
\hline \multirow{2}{*}{1.0} & 0.5 & 1.500315905 & 1.50184204 & 1.418619090 & 1.42356378 & 1.315714795 & 1.34008777 \\
\hline & 1.0 & 1.132515035 & 1.10213589 & 1.172791685 & 1.07700616 & 1.203327846 & 1.04262528 \\
\hline \multirow{2}{*}{1.5} & 0.5 & 1.926443755 & 1.92840335 & 1.821542968 & 1.82789208 & 1.689411238 & 1.72070676 \\
\hline & 1.0 & 1.454178089 & 1.41517050 & 1.505894331 & 1.38290328 & 1.545103502 & 1.33875737 \\
\hline
\end{tabular}

The value of $e_{3}(\rho)$ is approximated using a numerical integration. The minimal value of the function (37) is the value of the auxiliary term $\rho$. Here, for different values of $\alpha$, we get different values of auxiliary parameters using Maple software. For $\alpha=1, \alpha=0.9, \alpha=0.8, \alpha=0.7, \alpha=0.6$, the corresponding values of the auxiliary parameter $\rho$ are $1.21413823669280,0.461366433098273,1.29274273230225$, 1.33274646640909 , and 1.36918865275675 , respectively.

It is convenient to iterate many times to arrive at the given accuracy for the advanced computer technique, and the exact solution $\mathscr{U}(\psi)$ is obtained by the limit

$$
\mathscr{U}(\psi)=\lim _{k \longrightarrow \infty} \mathcal{U}_{k}(\psi) .
$$

The numerical solutions generated using the proposed technique with an optimal value of the auxiliary parameter $h=1.12170161455440$ are reported in Table 1. To show the applicability and efficiency of the proposed technique, the comparisons are carried out with the exact solutions as well as the standard variational iteration method from [60], in terms of absolute errors reported in Table 2 for different values of $t$ and $x$.

A full agreement between the results of fractional variational iteration algorithm and exact solution can be observed, which confirms applicability and accuracy of the proposed algorithm. One can observe that the proposed algorithm is very rapid, effective, and accurate, and this is proved by comparing the solutions obtained through the proposed method with the results in [60] as well as with the exact solution. Results are also shown graphically by assigning different values of $\alpha$ with $\psi=1$ under the given condition in each case, which can be seen in Figure 1.

The space and time surface graphs of approximate and exact solutions are observable in Figures 2-7.

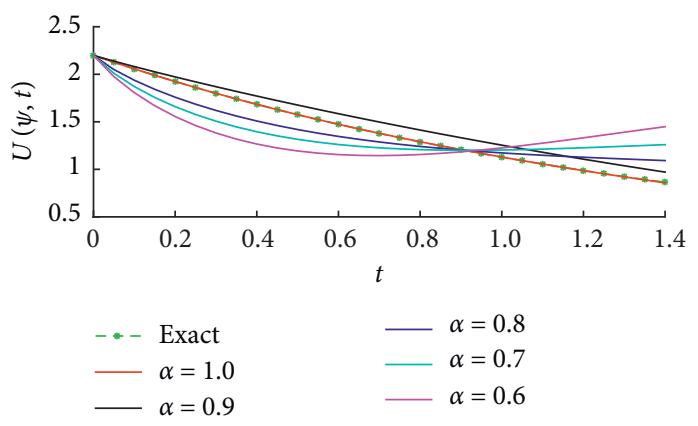

FIgURE 1: Plots of the approximate solution at $\psi=1$ for different values of $\alpha$.

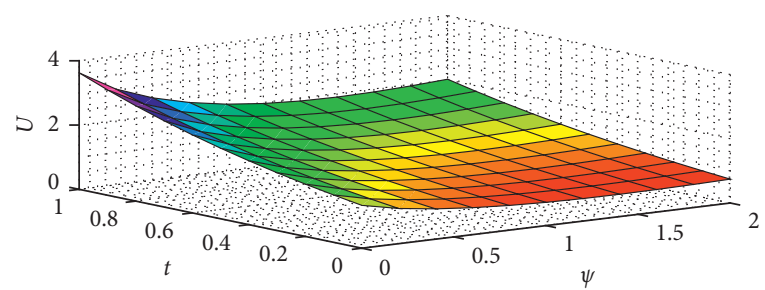

Figure 2: The surface graph of the approximated solution $\mathcal{U}_{3}(\psi, t, \rho)$ for $\alpha=0.6$.

\section{Discussion}

The nonlinear PDEs have become a hot topic in the field of nonlinear science and have been used in modeling miscellaneous problems in many fields of science and engineering, including plasma physics, fluid mechanics, quantum mechanics, fluid dynamics, image processing and economic system. However, for some complex problems in 


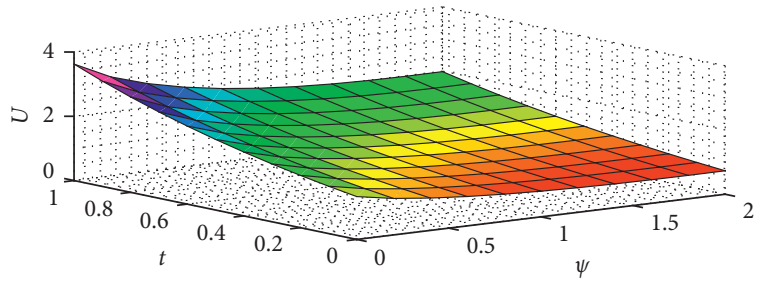

FIgURE 3: The surface graph of the approximated solution $\mathscr{U}_{3}(\psi, t, \rho)$ for $\alpha=0.7$.

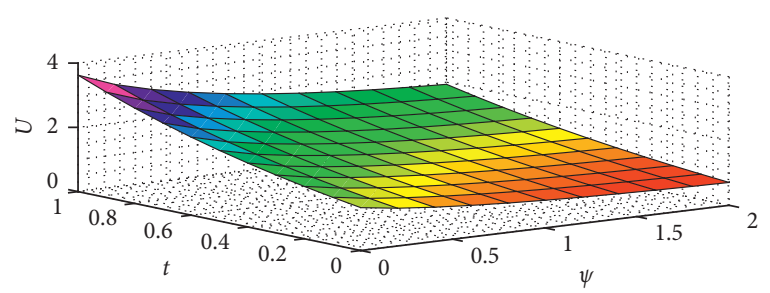

FIgURE 4: The surface graph of the approximated solution $\mathscr{U}_{3}(\psi, t, \rho)$ for $\alpha=0.8$.

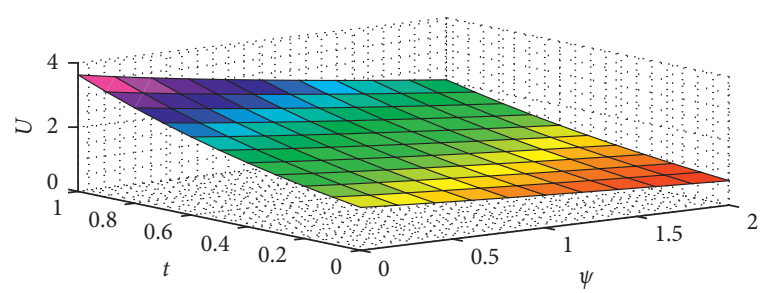

FIgURE 5: The surface graph of the approximated solution $\mathcal{U}_{3}(\psi, t, \rho)$ for $\alpha=0.9$.

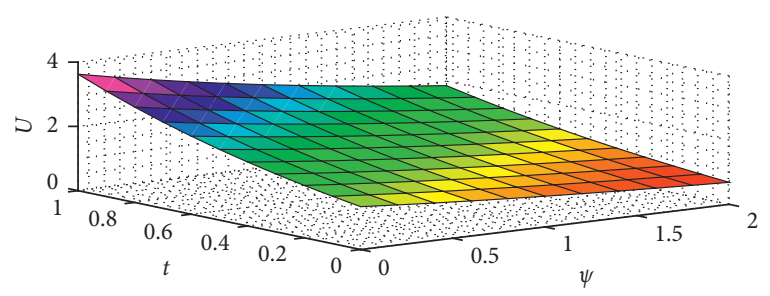

Figure 6: The surface graph of the approximated solution $\mathcal{U}_{3}(\psi, t, \rho)$ for $\alpha=1.0$.

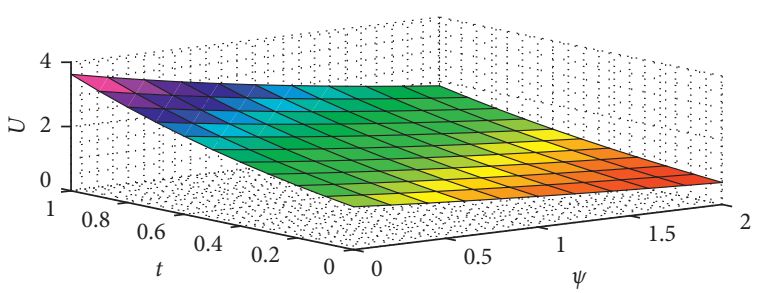

Figure 7: The behavior of the exact solution $\mathcal{U}(\psi, t, \rho)$.

these fields, the fractional partial differential equation is more accurate than integer partial differential equation. So, finding the numerical solutions of PDEs, as well as of fractional PDEs, has emerged as an extremely important strategy.

(1) Many engineering problems and other physical phenomena are modeled by nonlinear PDEs. On the other hand, the proposed iteration algorithms can be applied to various nonlinear PDEs. As a consequence, the proposed iterative scheme can be used in solving various problems.

(2) The fractional-order operator plays an important role not only in mathematics but also in other fields such as mechanics, physics, biomedical engineering, and finance. There are many nonlinear problems in real life. Therefore, there is a need to study the fractional PDEs.

(3) The numerical approaches are used for deeper understanding to predict the anomalies, which are not possible in the analytical methods because the analytical method can solve only two or three unknown variables, while numerical methods are applicable to much more variables very accurately.

(4) Analytical methods, if available, are always the best. However, these are impossible to achieve in some cases. If the analytical approaches are unable to generate the exact solution, numerical methods give the possibility to obtain an approximate numerical solution.

(5) The extreme benefit of the proposed algorithm is based on its straightforward applicability and conciseness. Furthermore, it can handle all types of nonlinear fractional PDEs.

\section{Conclusion}

In this work, a novel approach named as fractional iteration algorithm-I is introduced for various types of nonlinear PDEs as well as fractional PDEs and has been discussed in detail, including figures and tabulated numerical results. The proposed method is able to implement without the use of any transformation, linearization, discretization, or restrictive assumptions and thus is particularly perfect with the flexible and expanded nature of the physical problems. The coupling of Caputo fractional derivative and variational iteration algorithm-I to tackle both time and space derivatives accurately makes the solution process easiest, and the evaluation of fractional term becomes simpler, and FPDEs can be solved in a tremendous way. The proposed technique is able to solve all kinds of linear and nonlinear problems of physical nature arising in applied sciences and engineering.

\section{Data Availability}

Data will be provided on request to the first author.

\section{Conflicts of Interest}

The authors declare that there are no conflicts of interest associated with this publication. 


\section{Acknowledgments}

Predrag Stanimirović acknowledges support from the Ministry of Education, Science and Technological Development, Republic of Serbia, under Grant no. 174013. The research was supported by the National Natural Science Foundation of China (Grant nos. 11971142, 11871202, 61673169, 11701176, 11626101, and 11601485).

\section{References}

[1] X. Zhang, H. Zhu, and L.-H. Kuo, "A comparison study of the lmaps method and the ldq method for time-dependent problems," Engineering Analysis with Boundary Elements, vol. 37, no. 11, pp. 1408-1415, 2013.

[2] P. J. Harris, "The mathematical modelling of the motion of biological cells in response to chemical signals," in Computational and Analytic Methods in Science and Engineering, pp. 151-171, Springer, Berlin, Germany, 2020.

[3] H. Wang and N. Yamamoto, "Using a partial differential equation with google mobility data to predict COVID-19 in Arizona," Mathematical Biosciences and Engineering, vol. 17, no. 5, pp. 4891-4904, 2020.

[4] A. Viguerie, G. Lorenzo, F. Auricchio et al., "Simulating the spread of COVID-19 via a spatially-resolved susceptible-exposed-infected-recovered-deceased (SEIRD) model with heterogeneous diffusion," Applied Mathematics Letters, vol. 111, Article ID 106617, 2021.

[5] J. J. Ahmed, "Designing the shape of corona virus using the PDE method," General Letters in Mathematics, vol. 8, no. 2, pp. 75-82, 2020.

[6] R. Hilfer, Applications of Fractional Calculus in Physics, World Scientific Singapore, vol. 35, Singapore, 2000.

[7] I. Podlubny, Fractional Differential Equations: An Introduction to Fractional Derivatives, Fractional Differential Equations, to Methods of Their Solution and Some of Their Applications, Elsevier, Berlin, Germany, 1998.

[8] J.-H. He and Q.-T. Ain, "New promises and future challenges of fractal calculus: from two-scale thermodynamics to fractal variational principle," Thermal Science, vol. 24, no. 2 Part A, pp. 659-681, 2020.

[9] J.-H. He and H. Latifizadeh, "A general numerical algorithm for nonlinear differential equations by the variational iteration method," International Journal of Numerical Methods for Heat \& Fluid Flow, 2020.

[10] Y. Shen and Y. O. El-Dib, "A periodic solution of the fractional sine-gordon equation arising in architectural engineering," Journal of Low Frequency Noise, Vibration and Active Control, Article ID 146134842091756, 2020.

[11] Y. Jiang and J. Ma, "High-order finite element methods for time-fractional partial differential equations," Journal of Computational and Applied Mathematics, vol. 235, no. 11, pp. 3285-3290, 2011.

[12] Y. Liu, Z. Fang, H. Li, and S. He, "A mixed finite element method for a time-fractional fourth-order partial differential equation," Applied Mathematics and Computation, vol. 243, pp. 703-717, 2014.

[13] L. Qiu, W. Deng, and J. S. Hesthaven, "Nodal discontinuous galerkin methods for fractional diffusion equations on $2 \mathrm{~d}$ domain with triangular meshes," Journal of Computational Physics, vol. 298, pp. 678-694, 2015.

[14] F. Liu, P. Zhuang, I. Turner, K. Burrage, and V. Anh, "A new fractional finite volume method for solving the fractional diffusion equation," Applied Mathematical Modelling, vol. 38, no. 15-16, pp. 3871-3878, 2014.

[15] A. H. Bhrawy, "A Jacobi spectral collocation method for solving multi-dimensional nonlinear fractional sub-diffusion equations," Numerical Algorithms, vol. 73, no. 1, pp. 91-113, 2016.

[16] M. Dehghan, S. A. Yousefi, and A. Lotfi, "The use of He's variational iteration method for solving the telegraph and fractional telegraph equations," International Journal for Numerical Methods in Biomedical Engineering, vol. 27, no. 2, pp. 219-231, 2011.

[17] D. Lu, A. R. Seadawy, and M. M. Khater, "Structure of solitary wave solutions of the nonlinear complex fractional generalized Zakharov dynamical system," Advances in Difference Equations, vol. 2018, no. 1, p. 266, 2018.

[18] N. A. Shah, X. Wang, H. Qi, S. Wang, and A. Hajizadeh, "Transient electro-osmotic slip flow of an oldroyd-B fluid with time-fractional Caputo-Fabrizio derivative," Journal of Applied and Computational Mechanics, vol. 5, no. 4, pp. 779-790, 2019.

[19] J. Li, Y. Qiu, D. Lu, R. Attia, and M. Khater, "Study on the solitary wave solutions of the ionic currents on microtubules equation by using the modified Khater method," Thermal Science, vol. 23, no. Suppl. 6, pp. 2053-2062, 2019.

[20] R. M. Jena and S. Chakraverty, "Residual power series method for solving time-fractional model of vibration equation of large membranes," Journal of Applied and Computational Mechanics, vol. 5, no. 4, pp. 603-615, 2018.

[21] M. M. Khater, D. Lu, and R. A. Attia, "Dispersive long wave of nonlinear fractional $\mathrm{Wu}$-Zhang system via a modified auxiliary equation method," AIP Advances, vol. 9, no. 2, Article ID 025003, 2019.

[22] I. Ahmad, M. N. Khan, M. Inc, H. Ahmad, and K. S. Nisar, "Numerical simulation of simulate an anomalous solute transport model via local meshless method," Alexandria Engineering Journal, vol. 59, no. 4, pp. 2827-2838, 2020.

[23] M. N. Khan, I. Ahmad, and H. Ahmad, "A radial basis function collocation method for space-dependent inverse heat problems," Journal of Applied and Computational Mechanics, vol. 6, no. SI, pp. 1187-1199, 2020.

[24] M. Lakestani, M. Dehghan, and S. Irandoust-Pakchin, "The construction of operational matrix of fractional derivatives using B-spline functions," Communications in Nonlinear Science and Numerical Simulation, vol. 17, no. 3, pp. 11491162, 2012.

[25] J. Zhang and C. Xu, "Finite difference/spectral approximations to a water wave model with a nonlocal viscous term," Applied Mathematical Modelling, vol. 38, no. 19-20, pp. 4912-4925, 2014.

[26] Y. Lin, X. Li, and C. Xu, "Finite difference/spectral approximations for the fractional cable equation," Mathematics of Computation, vol. 80, no. 275, pp. 1369-1396, 2011.

[27] J.-H. He, "Approximate analytical solution for seepage flow with fractional derivatives in porous media," Computer Methods in Applied Mechanics and Engineering, vol. 167, no. 1-2, pp. 57-68, 1998.

[28] Z. Odibat and S. Momani, “The variational iteration method: an efficient scheme for handling fractional partial differential equations in fluid mechanics," Computers \& Mathematics with Applications, vol. 58, no. 11-12, pp. 2199-2208, 2009.

[29] M. Inc, "The approximate and exact solutions of the spaceand time-fractional Burgers equations with initial conditions by variational iteration method," Journal of Mathematical Analysis and Applications, vol. 345, no. 1, pp. 476-484, 2008. 
[30] R. Y. Molliq, M. S. M. Noorani, I. Hashim, and R. R. Ahmad, "Approximate solutions of fractional Zakharov-Kuznetsov equations by VIM," Journal of Computational and Applied Mathematics, vol. 233, no. 2, pp. 103-108, 2009.

[31] M. Safari, D. D. Ganji, and M. Moslemi, “Application of He's variational iteration method and Adomian's decomposition method to the fractional KdV-Burgers-Kuramoto equation," Computers \& Mathematics with Applications, vol. 58, no. 1112, pp. 2091-2097, 2009.

[32] S. Das, "Analytical solution of a fractional diffusion equation by variational iteration method," Computers \& Mathematics with Applications, vol. 57, no. 3, pp. 483-487, 2009.

[33] J. Lu, "An analytical approach to the Fornberg-Whitham type equations by using the variational iteration method," Computers \& Mathematics with Applications, vol. 61, no. 8, pp. 2010-2013, 2011.

[34] F. Abidi and K. Omrani, "The homotopy analysis method for solving the Fornberg-Whitham equation and comparison with Adomian's decomposition method," Computers \& Mathematics with Applications, vol. 59, no. 8, pp. 2743-2750, 2010.

[35] J. Zhou and L. Tian, "A type of bounded traveling wave solutions for the Fornberg-Whitham equation," Journal of Mathematical Analysis and Applications, vol. 346, no. 1, pp. 255-261, 2008.

[36] J. Yin, L. Tian, and X. Fan, "Classification of travelling waves in the Fornberg-Whitham equation," Journal of Mathematical Analysis and Applications, vol. 368, no. 1, pp. 133-143, 2010.

[37] C. Feng and $\mathrm{C}$. Wu, "The classification of all single traveling wave solutions to Fornberg-Whitham equation," International Journal of Nonlinear Science, vol. 7, no. 3, pp. 353-359, 2009.

[38] B. Jiang and Q. Bi, "Smooth and non-smooth traveling wave solutions of the Fornberg-Whitham equation with linear dispersion term," Applied Mathematics and Computation, vol. 216, no. 7, pp. 2155-2162, 2010.

[39] S. Hesam, A. Nazemi, and A. Haghbin, "Reduced differential transform method for solving the Fornberg-Whitham type equation," International Journal of Nonlinear Science, vol. 13, no. 2, pp. 158-162, 2012.

[40] J. Biazar and M. Eslami, "Approximate solutions for Fornberg-Whitham type equations," International Journal of Numerical Methods for Heat \& Fluid Flow, vol. 22, no. 6, pp. 803-812, 2012.

[41] B. Boutarfa, A. Akgül, and M. Inc, "New approach for the Fornberg-Whitham type equations," Journal of Computational and Applied Mathematics, vol. 312, pp. 13-26, 2017.

[42] S. Kumar, "An analytical algorithm for nonlinear fractional Fornberg-Whitham equation arising in wave breaking based on a new iterative method," Alexandria Engineering Journal, vol. 53, pp. 224-231, 2014.

[43] M. S. Al-luhaibi, "An analytical treatment to fractional Fornberg-Whitham equation," Mathematical Sciences, vol. 11, no. 1, pp. 1-6, 2017.

[44] A. A. Alderremy, H. Khan, R. Shah, S. Aly, and D. Baleanu, "The analytical analysis of time-fractional Fornberg-Whitham equations," Mathematics, vol. 8, no. 6, p. 987, 2020.

[45] I. Podlubny, "Fractional differential equations: an introduction to fractional derivatives, fractional differential equations, to methods of their solution and some of their applications," Mathematics in Science and Engineering, vol. 198, pp. 1-340, 1999.
[46] G. M. Mittag-Leffler, "Sur la nouvelle fonction $E_{\alpha}(x)$," Comptes Rendus De l'Academie Des Sciences Paris, vol. 137, pp. 554-558, 1903.

[47] G. Jumarie, "Modified Riemann-Liouville derivative and fractional Taylor series of nondifferentiable functions further results," Computers \& Mathematics with Applications, vol. 51, no. 9-10, pp. 1367-1376, 2006.

[48] M. Caputo, "Linear models of dissipation whose $Q$ is almost frequency independent--II," Geophysical Journal International, vol. 13, no. 5, pp. 529-539, 1967.

[49] A. Atangana and D. Baleanu, "New fractional derivatives with nonlocal and non-singular kernel: theory and application to heat transfer model," Thermal Science, vol. 20, no. 2, pp. 763-769, 2016.

[50] J.-H. He, "A tutorial review on fractal spacetime and fractional calculus," International Journal of Theoretical Physics, vol. 53, no. 11, pp. 3698-3718, 2014.

[51] J.-H. He, "Notes on the optimal variational iteration method," Applied Mathematics Letters, vol. 25, no. 10, pp. 1579-1581, 2012.

[52] H. Ghaneai and M. M. Hosseini, "Variational iteration method with an auxiliary parameter for solving wave-like and heat-like equations in large domains," Computers \& Mathematics with Applications, vol. 69, no. 5, pp. 363-373, 2015.

[53] H. Ahmad, "Variational iteration method with an auxiliary parameter for solving differential equations of the fifth order," Nonlinear science letters A, vol. 9, no. 1, pp. 27-35, 2018.

[54] D.-N. Yu, J.-H. He, and A. G. Garcia, "Homotopy perturbation method with an auxiliary parameter for nonlinear oscillators," Journal of Low Frequency Noise, Vibration and Active Control, vol. 38, p. 1461348418811028, 2018.

[55] H. Ahmad, T. A. Khan, H. Durur, G. M. Ismail, and A. Yokus, "Analytic approximate solutions of diffusion equations arising in oil pollution," Journal of Ocean Engineering and Science, 2020.

[56] M. Inokuti, H. Sekine, and T. Mura, "General use of the Lagrange multiplier in nonlinear mathematical physics," Variational Method in the Mechanics of Solids, vol. 33, no. 5, pp. 156-162, 1978.

[57] Z. M. Odibat, "A study on the convergence of variational iteration method," Mathematical and Computer Modelling, vol. 51, no. 9-10, pp. 1181-1192, 2010.

[58] H. Ghaneai and M. Hosseini, "Solving differential-algebraic equations through variational iteration method with an auxiliary parameter," Applied Mathematical Modelling, vol. 40, no. 5-6, pp. 3991-4001, 2016.

[59] H. Ahmad, A. R. Seadawy, and T. A. Khan, "Study on numerical solution of dispersive water wave phenomena by using a reliable modification of variational iteration algorithm," Mathematics and Computers in Simulation, vol. 177, pp. 13-23, 2020.

[60] M. G. Sakar, F. Erdogan, and A. Yıldırım, "Variational iteration method for the time-fractional Fornberg-Whitham equation," Computers \& Mathematics with Applications, vol. 63, no. 9, pp. 1382-1388, 2012. 